\title{
Distortion of crabbed bunch due to the electron cloud
}

\author{
L. Wang and T. Raubenheimer, SLAC
}

\begin{abstract}
In order to improve the luminosity, two crab cavities have been installed in KEKB HER and LER [1]. Since there is only one crab cavity in each ring, the crab cavity generates a horizontally titled bunch along the whole ring. The achieved specific luminosity with crabbed bunch is higher, but it is not as high as that from beam-beam simulation [2]. One of the suspicions is the electron cloud. The electron cloud in LER (positron beam) may distort the crabbed bunch and cause the luminosity drop. This note briefly estimates the bunch shape distortion due to the electron cloud in KEKB LER.
\end{abstract}




\section{Distortion of crabbed bunch due to the electron cloud}

L. Wang and T. Raubenheimer, SLAC

\section{INTRODUCTION}

In order to improve the luminosity, two crab cavities have been installed in KEKB HER and LER [1]. Since there is only one crab cavity in each ring, the crab cavity generates a horizontally titled bunch along the whole ring. The achieved specific luminosity with crabbed bunch is higher, but it is not as high as that from beam-beam simulation [2]. One of the suspicions is the electron cloud. The electron cloud in LER (positron beam) may distort the crabbed bunch and cause the luminosity drop. This note briefly estimates the bunch shape distortion due to the electron cloud in KEKB LER. The main parameters used in this note are summarized in Table I.

Table I: Main parameters of the beam (LER) and electron cloud

\begin{tabular}{lcc}
\hline \hline Voltage of crab cavity & $V$ & $1.4 \mathrm{MV}$ \\
Frequency of Crab cavity & $f_{R F}$ & $509 \mathrm{MHz}$ \\
Beam energy & $E$ & $3.5 \mathrm{GeV}$ \\
Circumference & $C$ & $3016 \mathrm{~m}$ \\
Transverse tune & $Q_{x}, Q_{y}$ & $45.506,43.570$ \\
Longitudinal tune & $Q_{s}$ & 0.0246 \\
Phase advance between Crab cavity and IP & $\Delta \phi_{x, C r a L_{-} I P}$ & $10.25 \times 2 \pi$ \\
Distance between Crab cavity and IP & $S_{C r a b_{-} I P}$ & $683.5 \mathrm{~m}$ \\
Half crossing angle at IP & $\theta_{x, I P}$ & $11 \mathrm{mrad}$ \\
Betatron function at crab cavity & $\beta_{x, \text { crab }}$ & $45 \mathrm{~m}$ \\
Betatron function at IP & $\beta_{x}^{*}$ & $1.5 \mathrm{~m}$ \\
Horizontal emittance & $\varepsilon_{x}$ & $17.7 \mathrm{~nm}$ \\
Vertical emittance & $\varepsilon_{y}$ & $0.266 \mathrm{~nm}$ \\
Average beam size & $\sigma_{x}, \sigma_{y}$ & $0.42 \mathrm{~mm}, 0.06 \mathrm{~mm}$ \\
beam size at IP & $\sigma_{x^{*}}$ & $0.163 \mathrm{~mm}$ \\
Half bunch length & $\hat{z}$ & $14 \mathrm{~mm}$ \\
Number of positrons per bunch & $N$ & $7.5 \times 10^{10}$ \\
Electron cloud density without solenoid & $\rho_{e}$ & $1.0 \times 10^{12} \mathrm{~m}^{-3}$ \\
Pinch factor & $f_{p}$ & 10 \\
\hline
\end{tabular}

\section{CLOSED ORBIT OF CRABBED BUNCH}

The closed orbit at location $s$ of the ring due to the kick of crab cavity is

$$
X(s)=\frac{\sqrt{\beta_{x}(s) \beta_{x, \text { crab }}}}{2 \sin \left(\pi Q_{x}\right)} \cos \left(\pi Q_{x}-\mid \phi_{x}(s)-\phi_{x, \text { crab }}\right) \theta_{x}^{\text {crab }}
$$


Where $\theta_{x}^{c r a b}$ is the horizontal kick from the crab cavity. When the cavity works at zero phase, the kick is given by

$$
\theta_{x}^{c r a b}=\frac{e V \sin \left(2 \pi f_{R F}(z-\hat{z}) / c\right)}{E} .
$$

Here $z$ is the distance from the bunch head. The cavity also can work at $\pi$ phase position where the sign of the kick changes comparing with zero phase case. Therefore, Eq. (2) represents both cases. If the phase advance between the crab cavity and interaction point (IP) satisfies

$$
\Delta \phi_{x, c r a b_{-} I P}=\phi_{x}^{*}-\phi_{x, c r a b}=n \pi+\frac{\pi}{2},
$$

then the closed orbit at IP is

$$
X^{*}=\frac{\sqrt{\beta_{x}^{*} \beta_{x, c r a b}}}{2} \theta_{x}^{c r a b}
$$

The half crossing angle (both electron and positron beam are titled) of the crabbed bunch at IP is

$$
\frac{d X^{*}}{d z}=\tan \left(\theta_{I P}\right)
$$

Since the crossing angle is small, the bunch at IP is titled by an angle

$$
\theta_{x, I P}=\frac{\pi e V f_{R F} \sqrt{\beta_{x}^{*} \beta_{x, c r a b}}}{E c} .
$$

From Eqs. $(1-2,6)$, the closed orbit is linearly proportional to the particle position inside the bunch:

$$
X(s) \approx \theta_{x}(s)(z-\hat{z})
$$

Where $\theta_{x}(s)$ is the tilted angle of the crabbed bunch at location $s$

$$
\theta_{x}(s)=\theta_{x, I P} \frac{\sqrt{\beta_{x}(s) / \beta_{x}^{*}}}{\sin \left(\pi Q_{x}\right)} \cos \left(\pi Q_{x}-\mid \phi_{x}(s)-\phi_{x, \text { crab }}\right) .
$$

Eq.(7) indicates that the synchrotron and betatron motion is strongly coupled for a crabbed bunch. The COD is zero at the bunch center and there is a maximum COD at the bunch head and tail. There is a small COD when Qx is close to half integer. Figure 1 shows the COD of the crabbed bunch within one betatron period. Because Qs is small, the particle distribution inside the bunch is frozen and only the bunch's tilted angle changes in this short period of time. Figure 2 shows the tilted angle of the crabbed bunch along the ring. The peak tilted angle is more than $35 \mathrm{mrad}$. There is a small tilted angle of $0.64 \mathrm{mrad}$ at the crab cavity. 
When the cavity's working phase is close to $\pi / 2$, the bunch center receives a dipole kick. Figure 3 shows the measured horizontal orbit distortion by a crab kick with a working phase close to $\pi / 2$ [3]. The dipole kick is $\theta_{x}^{\text {crab }} \approx e V / E$ in this case and the COD is given by Eq.(1). This COD differs from the title angle in Eq. (8) by a constant factor, which can be seen from Figure 2 and 3: there is a very similar distribution, although the optics is slightly different.

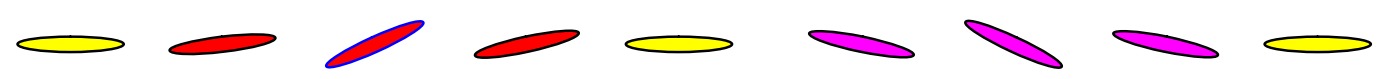

Figure 1: Snapshot of closed orbit in one betatron period

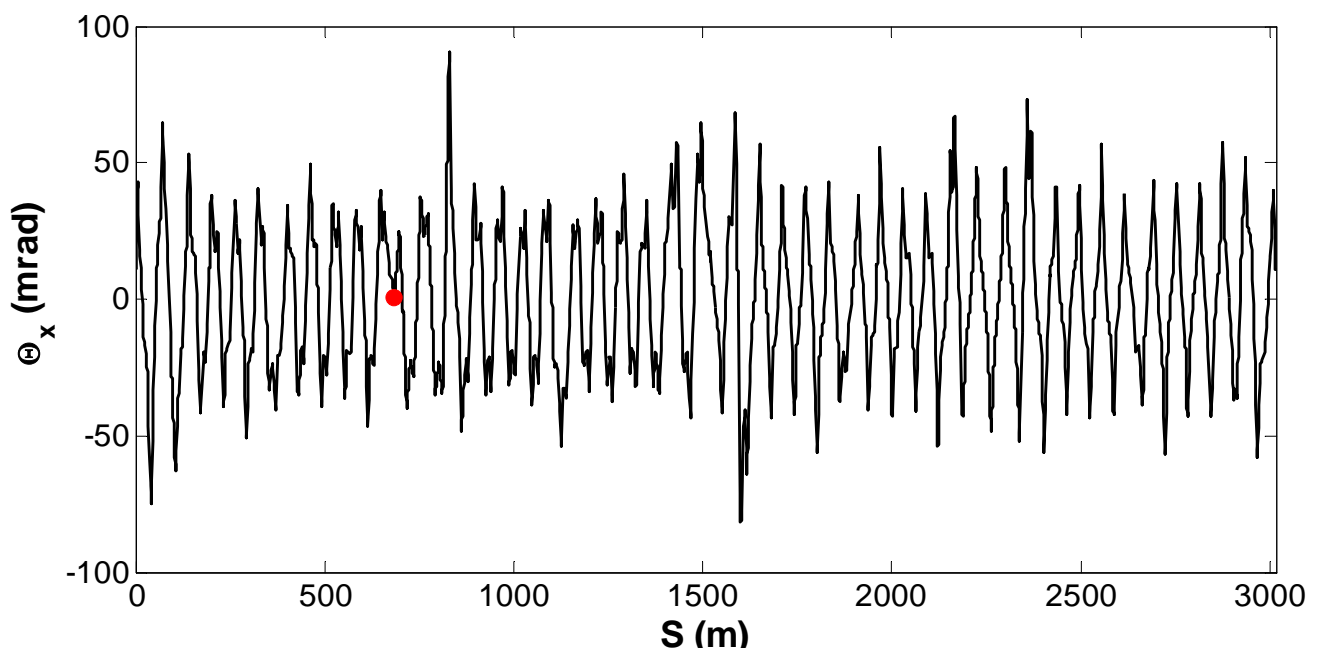

Figure 2: The titled angle of the crabbed bunch with realistic optics. It starts from the IP, and the crab cavity location is marked with the red dot.

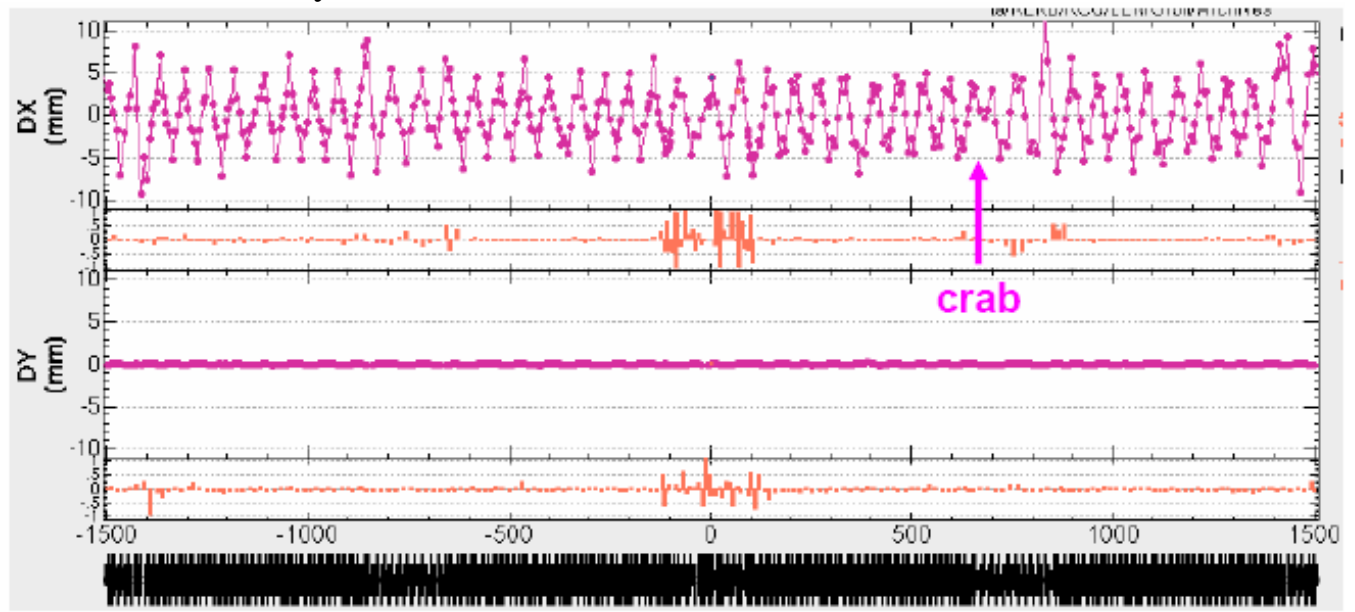

Figure 3: Horizontal orbit distortion ( $D x$ in the plot) by a crab kick measured with 453 BPMs. IP locates at $s=0$. The optics used in this plot is different from the one in this note. $\beta_{x}^{*}=0.8 \mathrm{~m}, \beta_{x, c r a b}=73 \mathrm{~m}, \mathrm{Qx}=45.505$. 


\section{BUNCH DISTORTION DUE TO THE ELECTRON CLOUD AT ONE SPECIFIC LOCATION}

\section{Wake function of electron cloud}

The linear space charge force between position bunch and electron cloud can be expressed by the wake function

$$
W=\frac{\gamma}{N r_{e}} \frac{\Delta x^{\prime}}{\Delta x}
$$

The wake field of space charge per unit length due to electron cloud is

$$
W(z)=P(z) \frac{\omega_{e}}{c} \frac{2 \hat{z}}{N} \frac{\lambda_{e}}{\sigma_{x}\left(\sigma_{y}+\sigma_{x}\right)} e^{-\frac{\omega_{e} z}{2 Q c}} \sin \left(\frac{\omega_{e} z}{c}\right) .
$$

Where $\lambda_{e}=2 \pi \rho_{e} \sigma_{x}^{2}, \rho_{e}$ is the electron density near bunch, The exponential decay of the wake is due to the nonlinear effect of the electron cloud. $P(z)$ is the enhancement factor due to beam pinch effect (both the density and size of electron cloud change with $z) . \omega_{\mathrm{e}}$ is the electron's bouncing frequency

$$
\omega_{e}=c\left(\frac{N r_{e}}{\hat{z}\left(\sigma_{x}+\sigma_{y}\right) \sigma_{x}}\right)^{1 / 2} .
$$

Figure 4 shows the simulated horizontal wake function due to the electron cloud for a titled bunch with tilted angle $11 \mathrm{mrad}$. Note that the wake shown in the figure is the wake due to the electron cloud along the whole ring. The simulated frequency of the wake function is $7.3 \times 10^{4} \mathrm{MHz}$, it is close to the calculated one from Eq. (11), $8.2 \times 10^{4} \mathrm{MHz}$. The $Q$ of the wake function is 4.5 . Therefore, the decay of the wake amplitude within one bunch length is negligible.

Due to the beam pinch effect, the electron cloud density is not a constant during the bunch passage as shown in Figure 5. The pinch factor $P(z)$ is the electron density normalized by the electron density at bunch head

$$
P(z)=\frac{\rho_{e}(z)}{\rho_{e}(0)}
$$

The pinch factor strongly depends on the bunch charge and bunch size. There is a peak pinch factor of 10 with the beam parameters listed in Table I. The strong pinch effect makes the bunch tail more unstable first and then the whole bunch becomes unstable due to synchrotron motion. 


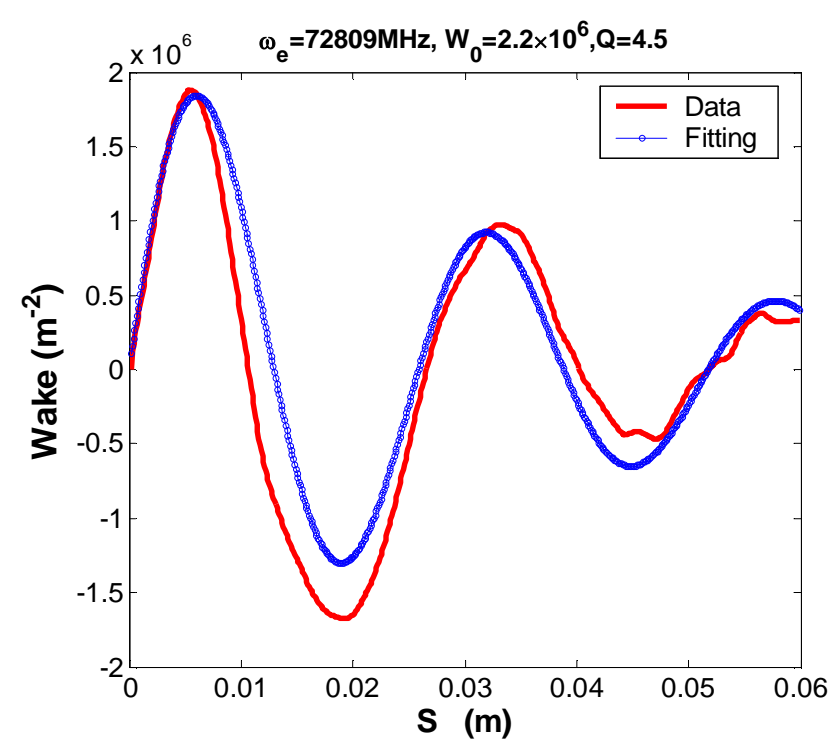

Figure 4: Horizontal wake due to the electron cloud, the bunch is titled by $11 \mathrm{mrad}$ in horizontal direction.

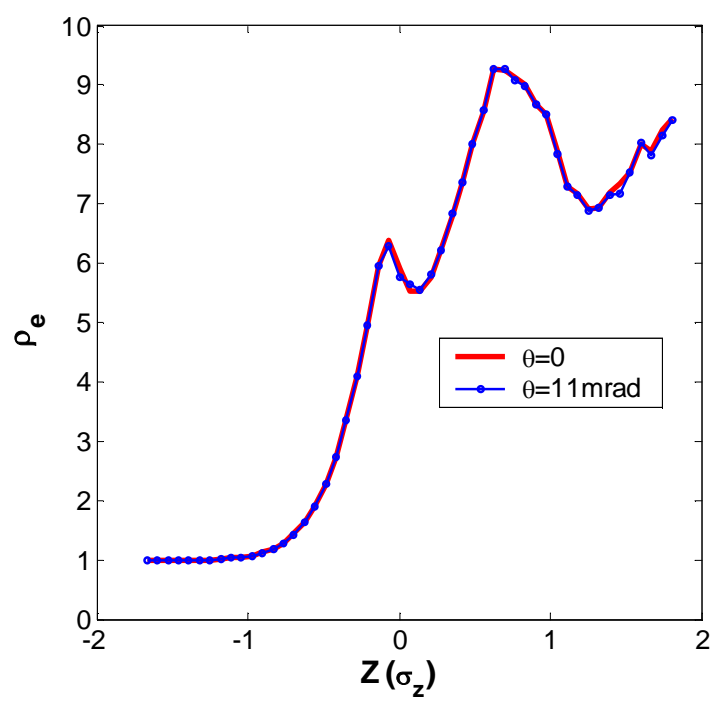

Figure 5: Variation of electron cloud density during the bunch passage. It is normalized by the initial electron cloud at the bunch head

\section{Distortion force}

At location $s$, the bunch has a tilted angle $\theta_{x}(s)$ (Eq.(8)). Following Chao's theory [4], the transverse kick received by a test charge at position $z$ due to the preceding particles is

$$
\Delta x^{\prime}(z)=\frac{N r_{e}}{\gamma} \int_{-\infty}^{z} d z^{\prime} \rho\left(z^{\prime}\right) x\left(z^{\prime}\right) W\left(z-z^{\prime}\right)
$$


For a uniform bunch

$$
\rho(z)= \begin{cases}\frac{1}{2 \hat{z}} & \text { if } 2 \hat{\mathrm{z}}>\mathrm{z}>0 \\ 0 & \text { otherwise }\end{cases}
$$

First let's consider a simple constant wake function (without considering the effects of beam pinch and electron oscillation around the bunch)

$$
W_{I}(z)=W_{0}=\frac{\omega_{e}}{c} \frac{\hat{z}}{N} \frac{4 \pi \rho_{e} \sigma_{x}}{\left(\sigma_{y}+\sigma_{x}\right)}
$$

Substituting $W$ and $\rho(z)$ into the equation for the kick force along the bunch yields

$$
\Delta x_{I}^{\prime}(s, z)=\frac{N r_{e} W_{0} \hat{z} \theta_{x}(s)}{\gamma}\left(\frac{z}{2 \hat{z}}\right)^{2}
$$

In the second wake model, we consider the electron oscillation but neglect the beam pinch effect $(P(z)=1)$ (drop the exponential decay part in Eq. (10))

$$
W_{I I}(z)=W_{0} \sin \left(\frac{\omega_{e} z}{c}\right)
$$

The corresponding kick force is

$$
\Delta x_{I I}^{\prime}(s, z)=\frac{N r_{e} W_{0} \hat{z} \theta_{x}(s)}{\gamma}\left(-2 \frac{z}{2 \widehat{z}} \alpha \cos \left(\omega_{e} z / c\right)+2 \alpha^{2} \sin \left(\omega_{e} z / c\right)\right)
$$

Where $\alpha=c /\left(2 \omega_{e} \hat{z}\right)$, which is the inverse of the electron oscillation number within one bunch length. It is close to 1 as shown in Figure 4 with the parameters in Table 1.

Due to the beam pinch effect, the density of the electron cloud near the bunch increases from the bunch head to tail as shown in Figure 5. To simplify the calculation, we assume $P(z)$ linearly increase with $z$ and there is a maximum factor of $f_{p}$ at the bunch tail

$$
P(z)=f_{p} \frac{z}{2 \hat{z}} .
$$

Then the wake function and the kick force are

$$
\begin{gathered}
W_{I I I}(z)=W_{0} \frac{f_{p} z}{2 \hat{z}} \sin \left(\frac{\omega_{e} z}{c}\right) \\
\Delta x_{I I I}^{\prime}(s, z)=\frac{N r_{e} W_{0} \hat{z} \theta_{x}(s)}{\gamma} f_{p}\left(-2\left(\frac{z}{2 \bar{z}}\right)^{2} \alpha \cos \left(\omega_{e} z / c\right)+4 \frac{z}{2 \bar{z}} \alpha^{2} \sin \left(\omega_{e} z / c\right)+4 \alpha^{3} \cos \left(\omega_{e} z / c\right)-4 \alpha^{3}\right)
\end{gathered}
$$

In summary, the kick force can be expressed as 


$$
\Delta x^{\prime}(s, z)=\frac{N r_{e} W_{0} \hat{z} \theta_{x}(s)}{\gamma} f_{p} F_{z}(z)
$$

Where

$$
F_{z}(z)= \begin{cases}\left(\frac{z}{2 \hat{z}}\right)^{2} & \text { (Mode I) } \\ -2 \frac{z}{2 \bar{z}} \alpha \cos \left(\omega_{e} z / c\right)+2 \alpha^{2} \sin \left(\omega_{e} z / c\right) & \text { (Mode II) } \\ -2\left(\frac{z}{2 \bar{z}}\right)^{2} \alpha \cos \left(\omega_{e} z / c\right)+4 \frac{z}{2 \bar{z}} \alpha^{2} \sin \left(\omega_{e} z / c\right)+4 \alpha^{3} \cos \left(\omega_{e} z / c\right)-4 \alpha^{3} & \text { (Mode III) }\end{cases}
$$

$f_{p}=1$ for model I and II. $F_{z}$ has a maximum 1 at the bunch tail for the constant wake model. It represents the shape of the distorted bunch as late shown. Figure 6 shows distortion factor $F z$ for different wake models. The constant wake model causes a larger distortion than the model II. There is similar distortion shape for Model II and III, but note that the factor $\boldsymbol{f p}$, which is about 10 for KEKB, is not included in the plot. Therefore, there likely is a largest distortion when the beam pinch effect (Model III) is included. It is interesting that the distortion monotonously increases with $z$ and bunch intensity $N$ when $\alpha>0.5$ (there is a smaller $\alpha$ for a higher intensity bunch). But when $\alpha<0.2$, the distortion starts to oscillate along the bunch.

With the given parameters in Table I, $\alpha$ is close 1 . Therefore, the distorted bunch has a banana shape. When the beam becomes strong enough $(\alpha<0.2)$ (with a larger bunch intensity or small beam size), the distorted bunch will have snake shape. Because of the dependence of $\alpha$ on the beam size, the distortion force varies along the ring. With the realistic optics, the calculated $\alpha$ ranges from 0.15 to 0.3 in most of the ring. Thence the distorted bunch may have a shape similar as the pink line in Figure 6. 


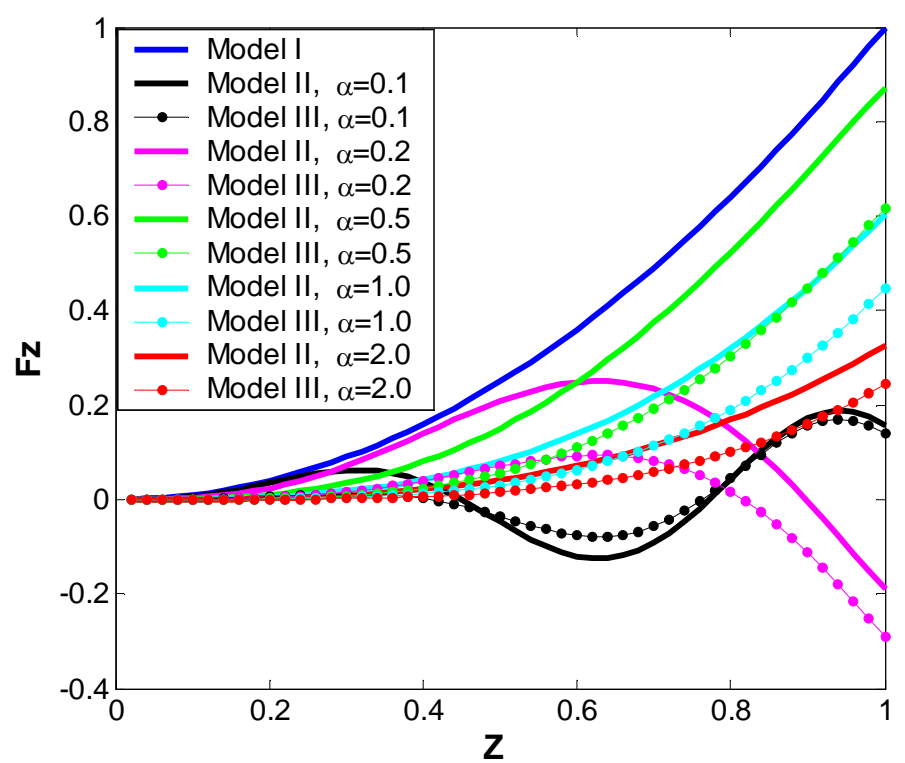

Figure 6: Distortion a long the bunch for different wake models and beam strength factor $\alpha$

Figure 7 shows the electron cloud kick along the ring with realistic optics. It has an amplitude of $8 \times 10^{-5} \mathrm{mrad} / \mathrm{m}$ and is in phase with the titled angle of the crabbed bunch shown in Figure 2.

Assuming all the electron cloud stays at one location $s$ and $F(z)=1$, the total kick value of $\Delta \mathrm{x}^{\prime}$ is

$$
\Delta x^{\prime}=\frac{N r_{e} W_{0} \hat{z} C}{\gamma} f_{p} \theta_{x}(s)
$$

With the given parameters, the total kick is about $7.46 \times 10^{-4} \theta(\mathrm{s})$, which is negligible. 


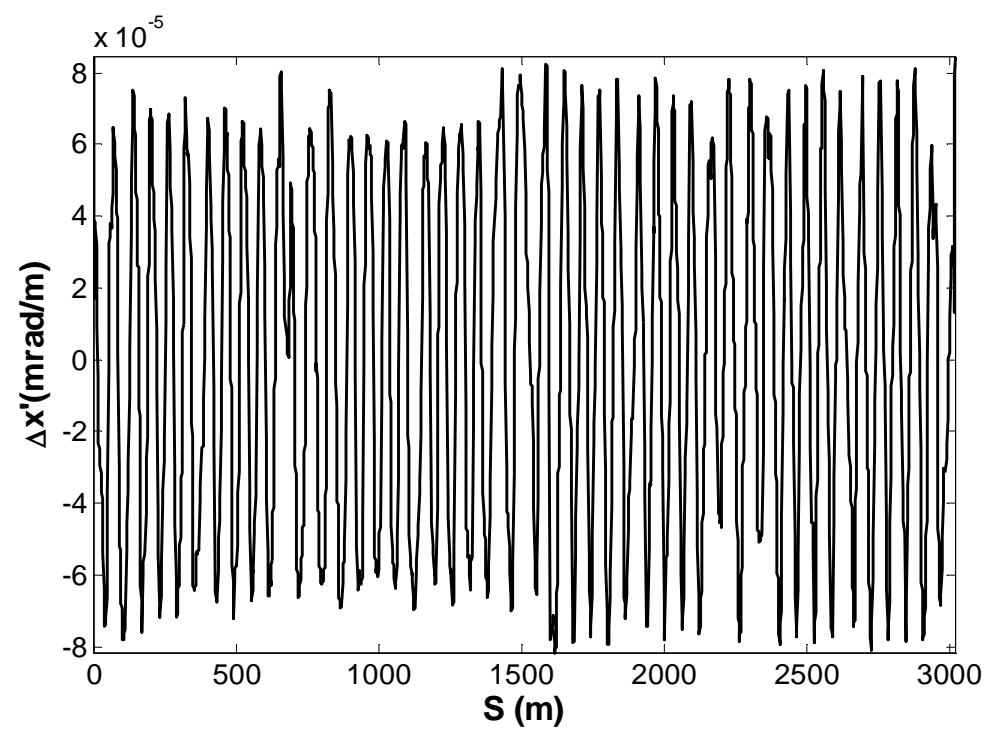

Figure 7: Estimated kick from electron cloud

\section{BUNCH DISTORTION DUE TO THE ELECTRON CLOUD IN THE WHOLE RING}

We assume the electron cloud is uniformly distributed along the ring. The COD due to electron cloud becomes:

$$
\Delta x(s, z)=\frac{\sqrt{\beta_{x}(s)}}{2 \sin \left(\pi Q_{x}\right)} \int_{0}^{c} d s^{\prime} \sqrt{\beta_{x}\left(s^{\prime}\right)} \cos \left(\pi Q_{x}-\left|\phi_{x}(s)-\phi_{x}\left(s^{\prime}\right)\right|\right) \Delta x^{\prime}\left(s^{\prime}, z\right)
$$

Substituting Eqs. (8) and (22) into the above equation, the COD at location s due to the electron cloud in the whole ring becomes

$$
\begin{aligned}
\Delta x(s, z)= & \frac{\beta_{x}(s) \theta_{x, I P}}{2 \sin ^{2}\left(\pi Q_{x}\right) \sqrt{\beta_{x}^{*}}} \frac{N r_{e} W_{0} \hat{z} f_{p} F(z)}{\gamma} \\
& \int_{0}^{c} d s^{\prime} \sqrt{\beta_{x}\left(s^{\prime}\right)} \cos \left(\pi Q_{x}-\left|\phi_{x}(s)-\phi_{x}\left(s^{\prime}\right)\right|\right) \cos \left(\pi Q_{x}-\left|\phi_{x}\left(s^{\prime}\right)-\phi_{x, \text { crab }}\right|\right)
\end{aligned}
$$

To simplify the calculations, a constant beta function $\bar{\beta}_{x}=\sqrt{C /\left(2 \pi Q_{x}\right)}$ is assumed, then the COD becomes

$$
\Delta x(s, z)=A(s) F_{s}(s) F_{z}(z)
$$

Where $A(s)$ gives the amplitude of the distortion

$$
A(s)=\frac{\beta_{x}(s) \theta_{x, I P}}{4 \sin ^{2}\left(\pi Q_{x}\right) \sqrt{\beta_{x}^{*}}} \frac{N r_{e} W_{0} C \hat{z} f_{p}}{\gamma} \sqrt{\bar{\beta}_{x}},
$$

The average $\mathrm{A}=0.57 \mathrm{~mm}$. Fs expresses the betatron phase effect, 


$$
\begin{aligned}
F_{s}(s)= & \frac{C-\left|s-s_{c r a b}\right|}{C} \cos \left(\phi_{x}(s)-\phi_{x, \text { crab }}\right)+\frac{\left|s-s_{c r a b}\right|}{C} \cos \left(2 \pi Q_{x}-\left|\phi_{x}(s)-\phi_{x, c r a b}\right|\right)+ \\
& \frac{2 \bar{\beta}_{x}}{C} \sin \left(\pi Q_{x}\right) \cos \left(\pi Q_{x}-\left|\phi_{x}(s)-\phi_{x, c r a b}\right|\right)
\end{aligned}
$$

At the crab cavity,

$$
F_{s}\left(s_{c r a b}\right)=1+\frac{\bar{\beta}_{x}}{C} \sin \left(2 \pi Q_{x}\right)
$$

Since $\bar{\beta}_{x}<<C$ for a large ring like KEKB, therefore, $F_{s}\left(s_{c r a b}\right)$ is close to 1 .

At IP

$$
\begin{aligned}
F\left(s_{I P}\right)= & \frac{C-\left(s_{c r a b}-s_{i p}\right)}{C} \cos \Delta \phi_{x, c r a b_{-} I P}+\frac{s_{c r a b}}{C} \cos \left(2 \pi Q_{x}-\Delta \phi_{x, c r a b_{-} I P}\right)+ \\
& \frac{2 \bar{\beta}_{x}}{C} \sin \left(\pi Q_{x}\right) \cos \left(\pi Q_{x}-\Delta \phi_{x, c r a b_{-} I P}\right)
\end{aligned}
$$

Using Eq. (3), the above equation becomes

$$
F\left(s_{I P}\right)= \pm\left[\frac{s_{c r a b}}{C} \sin \left(2 \pi Q_{x}\right)+\frac{2 \bar{\beta}_{x}}{C} \sin ^{2}\left(\pi Q_{x}\right)\right] \quad(+, \text { if } n \text { is even; }- \text { if } n \text { is odd })
$$

A $Q_{x}$ close to half integer is chosen in KEKB in order to get a high luminosity

$$
Q_{x}=m+0.5 .
$$

Substituting Eqs. (3) and (33) into Eq.(30) and (32), then

$$
\begin{gathered}
F_{s}\left(s_{\text {crab }}\right)=1 \\
F_{s}\left(s_{I P}\right)= \pm \frac{2 \bar{\beta}_{x}}{C}
\end{gathered}
$$

Figure 8 shows $F s$ along the ring starting from IP. It has a maximum about 1.0 at crab cavity. Very luckily, there is a very small $F_{s}\left(s_{I P}\right)$ of 0.0118 because of the half integer tune $Q_{x}$ and the special phase advance between the crabbed cavity and IP. The betatron tune dependence of $F_{s}$ at IP is shown in Figure 9. There is minimum of $F_{s}\left(s_{I P}\right)$ when $Q_{x}$ is close to half integer (Eq.32). Figure 10 shows $F_{s}(s)$ along the ring for different Qx. The overall shape varies with Qx.

In the above estimation, a uniform electron cloud is assumed. Now let's assume the electron cloud locates at some specific locations, the COD at IP due to the electron cloud at these specific locations is

$$
\Delta x(0, z)=\frac{\sqrt{\beta_{x}^{*}} \theta_{x, I P}}{2 \sin ^{2}\left(\pi Q_{x}\right)} \frac{N r_{e} W_{0} \hat{z} f_{p} F(z)}{\gamma} \sum_{i} \sqrt{\beta_{x}\left(s_{i}\right)} L_{i} \cos \left(\pi Q_{x}-\phi_{x}\left(s_{i}\right)\right) \cos \left(\pi Q_{x}-\mid \phi_{x}\left(s_{i}\right)-\phi_{x, \text { crab }}\right)
$$

Where $L_{i}$ the length of each section. Using Eqs.(3) and (33), it can be simplified as 


$$
\Delta x(0, z)= \pm \frac{\sqrt{\beta_{x}^{*}} \theta_{x, I P}}{4} \frac{N r_{e} W_{0} \hat{z} f_{p} F(z)}{\gamma} \sum_{i} \sqrt{\beta_{x}\left(s_{i}\right)} L_{i} \sin \left[2 \phi_{x}\left(s_{i}\right)\right] .
$$

Where the sign depends on $n$ in Eq.(3): ' + ' if $n$ is odd and ' - ' if $n$ is even. Since there are many electron cloud sections in the ring, the average effects on $C O D$ at IP should be small due to their cancellation (phase factor in the above equation). Therefore, the distortion at IP is likely small due to the conditions Eqs.(3) and (33). In another words, there is a small $F_{s}(0)$ when $Q_{x}$ is close to half integer, even with a non-uniform distributed electron cloud.

Figure 10 shows the COD along the ring with the constant betatron function model and a realistic optics. The overall shape agrees well except some fluctuations due to the variation of the betatron function with realistic optics. The COD at IP is 2.4 $\mu m$, which is about $1.5 \%$ of the bam size at IP $\left(\sigma_{\mathrm{x}}^{*}=0.163 \mathrm{~mm}\right)$.

The COD with different electron distribution along the ring is shown in Figure 11. The overall distribution of COD doesn't change much with a random electron distribution. It does change a lot when the electron cloud is far from uniform or random distribution. But there is always a small COD at IP, which implies that the COD at IP is always small no matter how the electron cloud is distributed along the ring. This agrees with Eq.(37).

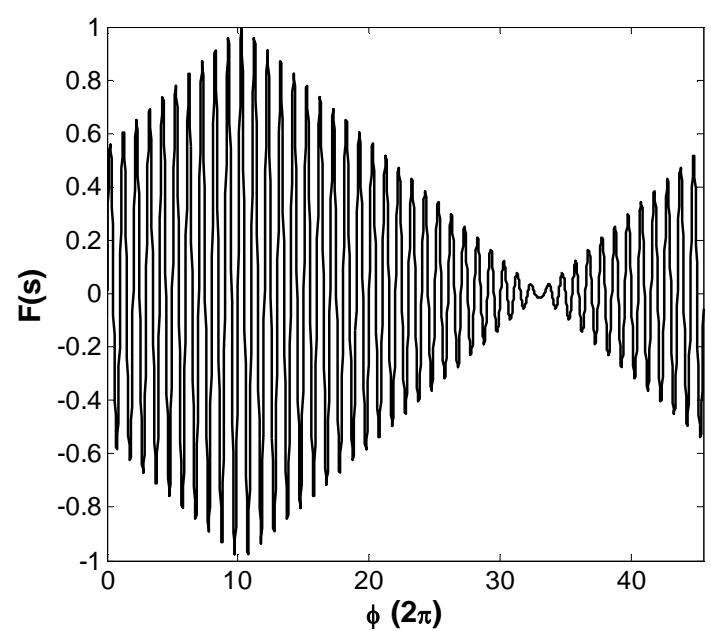

Figure 8: $\mathrm{F}(\mathrm{s})$ vs. the phase advance starting from IP. There is a maximum of 1.0 at the crab cavity. 

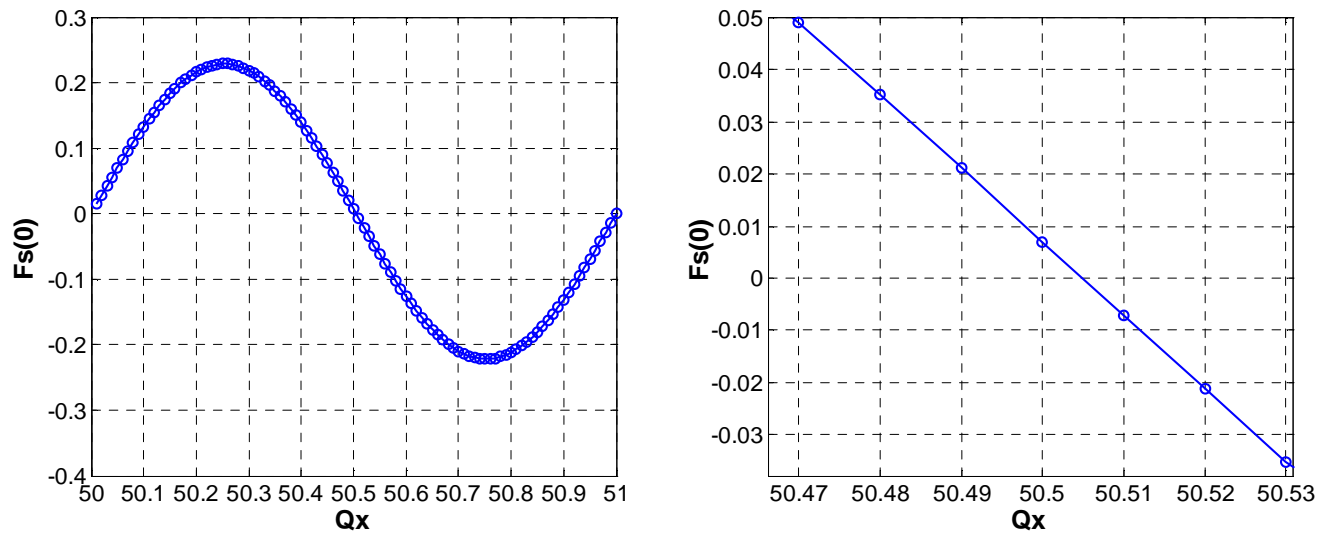

Figure 9: Dependence of $F s\left(\mathrm{~s}_{\mathrm{IP}}\right)$ on $\mathrm{Qx}$
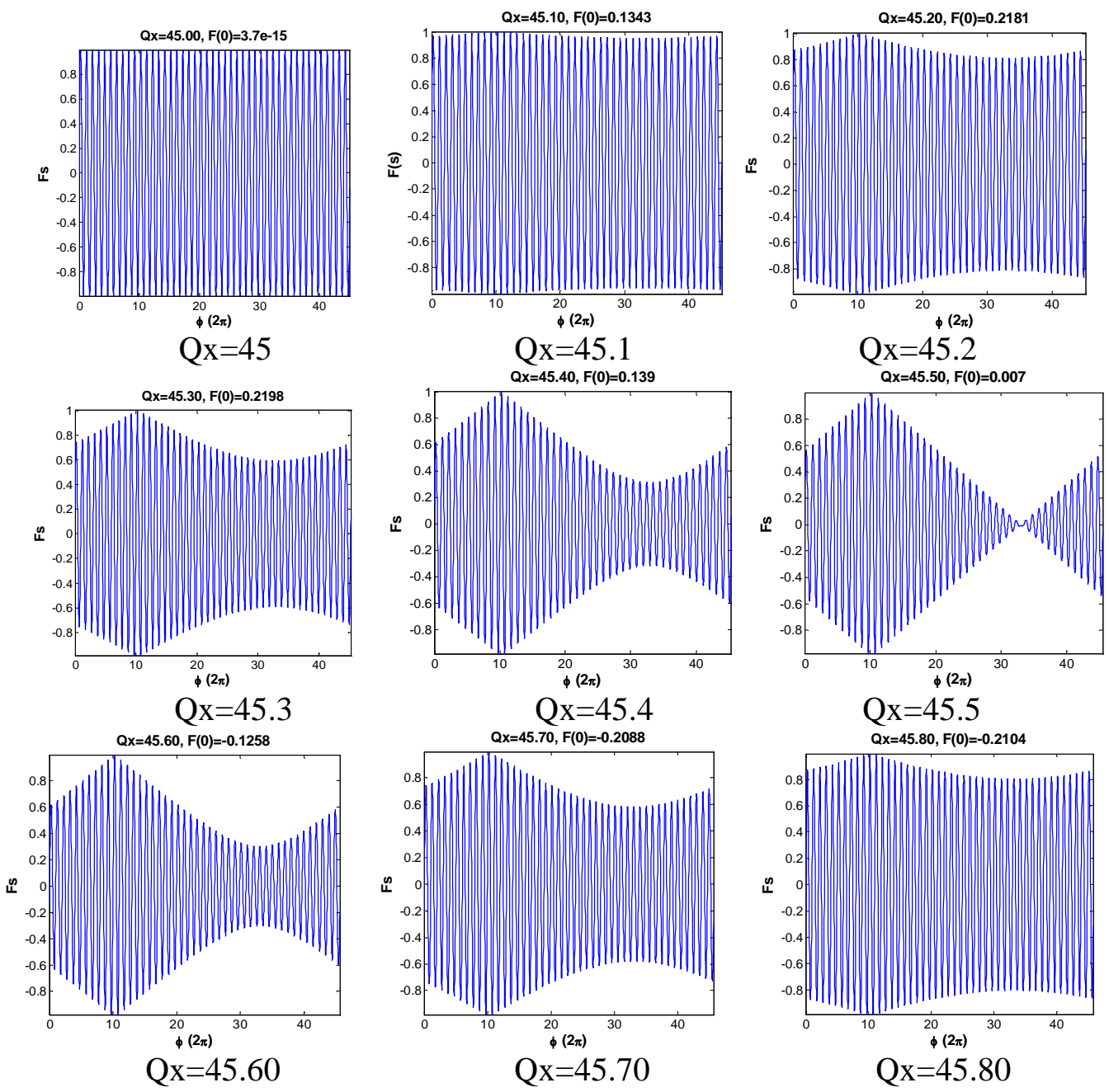

Figure 10: $\boldsymbol{F s}$ with different Qx 


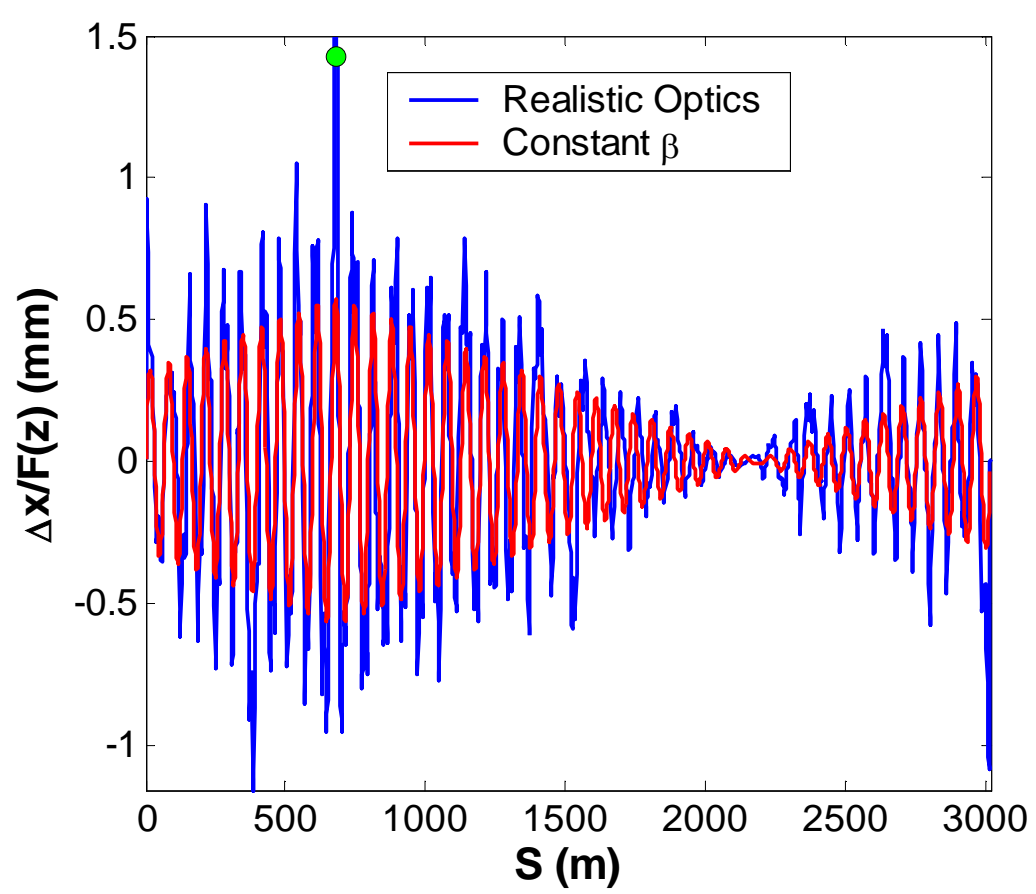

Figure 11: COD due to the electron cloud with analytical model and realistic optics. The location of crab cavity is marked with green dot. $f_{p}=10$.

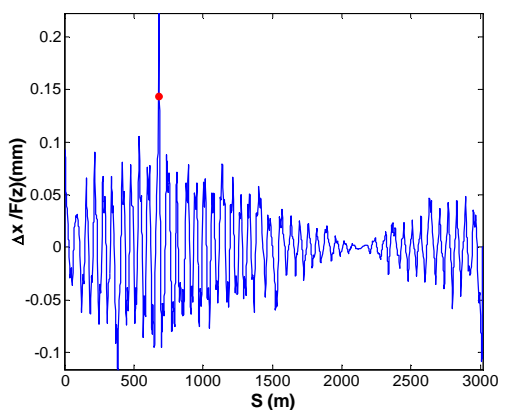

(a) uniform $\Delta X^{*}=0.54 \mu \mathrm{m}$

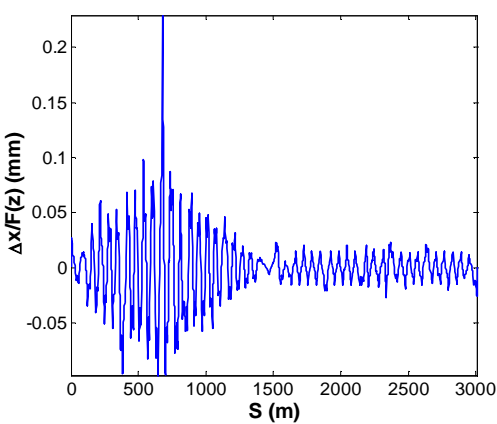

(c ) first half ring, uniform, $\Delta X^{*}=0.92 \mu \mathrm{m}$

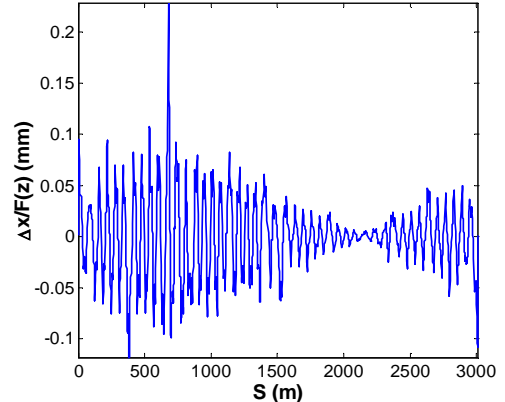

(b) random, $\Delta X^{*}=0.90 \mu \mathrm{m}$

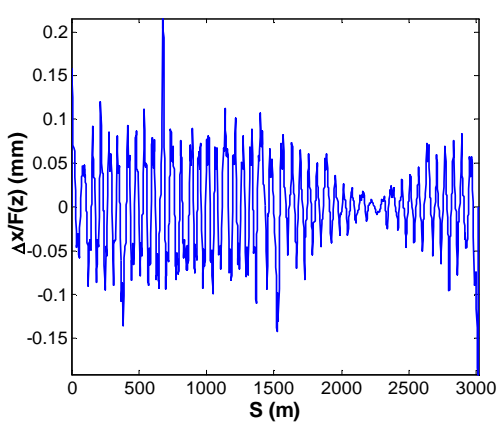

(d) second half ring, uniform, $\Delta X^{*}=0.16 \mu \mathrm{m}$

Figure 12: COD due to electron cloud with different distributions along the ring $\left(f_{p}=1.0\right)$. The ring starts from IP and the red dot shows the location of crab cavity. 


\section{DISCUSSION AND SUMMARY}

The horizontal orbit distortion by the crab cavity is naturally coupled with the synchrotron motion (Eq. 7). The induced synchrotron-betatron motion may be a concern.

The titled angle of the crabbed bunch a long the ring differs from the closed orbit when the cavity works at $\pi / 2$ by a constant factor. Therefore, the titled angle of the crabbed bunched can be indirectly measured by using this closed orbit. Since there are many BPMs, the titled bunch in the whole ring can be easily measured. This method also can be used to benchmark with the direct measurement [5].

The shape of the distorted bunch $(F z)$ due to electron cloud depends on the bunch line density and beam size $(\alpha)$. It has a banana shape with a weak beam, for instance $\alpha>0.5$. And it becomes a snake shape when the beam force is strong enough, $\alpha \sim 0.1$. With the present parameters in KEKB LER, the distorted bunch has a banana shape.

The titled angle of the crabbed bunch a long the ring (except IP) is inversely proportional the square root of $\beta_{x}^{*}$ (Eq.8). Therefore, a larger $\beta_{x}^{*}$ can reduce the distortion and other effects due to the crabbed bunch, if any.

The half integer betatron tune $Q_{x}$ (required for luminosity) and the specific phase advance between the crab cavity and IP (required to generate crabbed bunch) causes a small distortion at IP if there are many electron cloud sections in the ring. With a uniform distributed electron cloud, the distortion at IP $x^{*} / \sigma_{x}^{*}$ is about $0.015(2.4 \mu \mathrm{m})$. The change of luminosity due to this offset is small [1]. According to the simulation, the horizontal offset must be less than $20 \mu \mathrm{m}$ to see the luminosity enhancement by the crab crossing. Therefore, the small distortion due to electron cloud likely couldn't cause clear luminosity drop.

Note that the distortion at IP is sensitive to the horizontal tune. Near half integer, the distortion force is linearly depends on the distance from half integer and its sign changes when $\mathrm{Q}_{x}$ crosses the half integer.

In the above estimations, we assume an electron density of $1.0 \times 10^{12} \mathrm{~m}^{-3}$ with the solenoid off and a pinch factor $f_{p}$ of 10 is used, which is from simulation. Therefore, this could be the worst case. 
The KEKB experiment study shows that the solenoid can reduce the beam instability growth rate by a factor of 5 and 10 in horizontal and vertical direction, respectively. Therefore, one may expect a smaller distortion at IP with solenoid. However, one should be careful to draw the conclusion. The most important conclusion of this study is that the small distortion at IP benefits from the cancellation of electron cloud kicks in the whole ring (Eq.37). Reducing the number of electron cloud sections, for instance, with solenoid on, doesn't necessarily reduce the distortion at IP by the same factor. When the number of electron cloud section is small, the location of the electron cloud plays an important role. Likely, this number is still large even with solenoid on. Otherwise, the locations of electron cloud should be specified in order to check their contributions.

There is no distortion observed in the experiment [5], which probably can be explained by the negligible distortion according to the calculation here.

\section{ACKNOWLEDGEMENT}

We thank Dr. H. Fukuma for helpful discussion and kindly providing of the information and data.

\section{REFERENCE}

[1] T. Abe, et. al., in the proceedings of PAC07, TUPAN045, 2007

[2]Y. Funakoshi, et. al., in the proceedings of PAC07, THPAN037, 2007

[3]K. Ohmi, Beam-beam workshop, 2-4, July, 2007, SLAC

[4] A. W. Chao, Physics of Collective Beam Instabilities in High Energy Accelerators (Wiley, New York, 1993).

[5]H. Ikeda, et. al., in the proceedings of PAC07, FRPMN035, 2007 